\title{
Relations between Safety Climate, Awareness, and Behavior in the Chinese Construction Industry: A Hierarchical Linear Investigation
}

\author{
Mudan Wang $\mathbb{B D}^{1}{ }^{1}$ Jun Sun, ${ }^{1}$ Hua Du $\left(\mathbb{D},{ }^{1}\right.$ and Cynthia Wang ${ }^{2}$ \\ ${ }^{1}$ School of Civil Engineering and Mechanics, Huazhong University of Science and Technology, Wuhan, Hubei 430074, China \\ ${ }^{2}$ Faculty of Built Environment, The University of New South Wales, Sydney, NSW 2052, Australia \\ Correspondence should be addressed to Hua Du; duhua9610@163.com
}

Received 15 April 2018; Accepted 27 May 2018; Published 5 July 2018

Academic Editor: Eric Lui

Copyright (C) 2018 Mudan Wang et al. This is an open access article distributed under the Creative Commons Attribution License, which permits unrestricted use, distribution, and reproduction in any medium, provided the original work is properly cited.

\begin{abstract}
The vast majority of accidents in construction are generated by unsafe behaviors. Some researches also find that the behaviors could be influenced by the awareness and safety climate. The safety behavior and awareness belong to individual levels, while the safety climate belongs to the organization level. Previous studies mainly focus on the relationships between safety climate, safety awareness, and safety behavior without considering their different respective levels and the interaction between levels. This study establishes a hierarchical linear model (HLM) of safety climate, individual safety awareness, and safety behavior to examine the multilevel relationships between them. Data were collected using questionnaire from workers in different teams on the construction site in China. The results indicate that organizational safety climates affect individual safety behavior and safety awareness. In addition, there is a positive correlation between individual safety awareness and safety behavior, and the safety climates have a positive moderating effect on the relationship between them. The final conclusion offers a path for the current practice of safety management in the construction industry.
\end{abstract}

\section{Introduction}

Statistics show that the construction industry is still facing safety problems, which is well worth studying. Figure 1 shows the number of construction accidents and deaths declining respectively since 2004 according to MOHURD (Ministry of Housing and Urban-Rural Development) statistics in China. This shows that the number of the construction accidents and the annual death toll has decreased year by year; nevertheless, there are still many accidents which have led to tremendous losses. Therefore, the situation is still serious and needs to improve.

An analysis of the causes of construction accidents in China shows that unreasonable management, illegal operations, and labor indiscipline are responsible for more than $70 \%$ of the total number of accidents. It reveals that the vast majority of accidents are caused by people, which means that the risk of accidents and injuries can be reduced by safety behavior $[1,2]$. Meanwhile, the individual safety behavior could be improved by increasing the self-awareness of safety [3]. Niskanen [4] points out that attributing accidents solely exaggerates the influence of personal factors, underestimates the effect of environmental factors on individual behavior, and makes a scapegoat of anyone associated with the accident. In other words, the behavior of individuals may be the result of certain environmental factors in the organization, such as safety climate. Therefore, in order to reduce the construction accidents, both individual factors and organizational factors should be taken into consideration.

This paper focuses on the safety management in construction, aiming to improve the current situation. From the individual and organizational perspective, it is significant to analyze the relationship between safety climate, safety awareness, and safety behavior. The hierarchical linear model (HLM) is used to explore the influences that the safety climate has on safety awareness and safety 


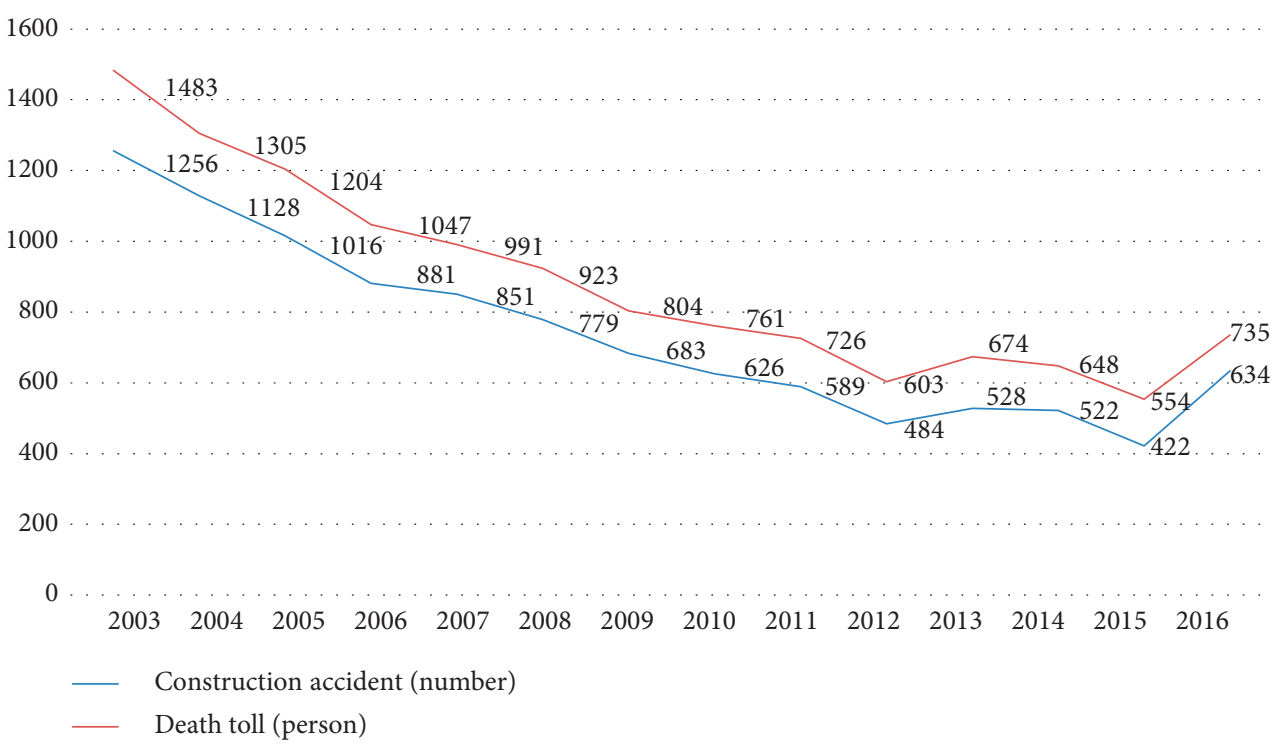

Figure 1: Construction accident statistics in China from 2003 to 2016.

behavior. Suggestions are put forward to improve safety management.

\section{Materials and Methods}

2.1. Safety Climate. In the construction industry, safety climate is often described as the organizational members' perceptions of the value placed on safety by management [5] and is regarded as a manifestation of safety culture in the behavior and expressed attitude of employees [6]. Safety climate comes from a bottom-up perceptional approach, concerned with worker perceptions of safety in the workplace [7]. Fang et al. [8] provide that the safety climate is a perception of the state of safety at a particular time and closely concerned with intangible issues such as environmental and situational factors. Furthermore, the safety climate is a reliable indicator among a variety of quantitative and qualitative data collection tools to measure the safety culture [9] and is concerned with the shared perceptions and beliefs that workers hold regarding safety in their workplace [10], including both physical and psychosocial safety climate $[11,12]$.

The safety climate has different ways of division on its dimensions according to the previous studies. Bosak et al. [13] put forward that safety climate has three dimensions, that is, management commitment to safety, priority of safety, and pressure for production, while Li et al. [14] believe that safety climate has six dimensions, that is, workers' self-perception of safety, workers' involvement in safety, co-workers' interaction, safety environment, safety management involvement, and safety personnel support. Other research identifies five critical dimensions of safety climate, which are management's commitment, safety behavior and employee involvement, incentives and rewards systems, communication and information systems, and work pressure [15].

The measurement items for safety climate varied in previous studies. As such, there is no consensus about the factor structure of the safety climate [7, 16]. Zohar and Luria's [17] organizational-level safety climate scale consisted of a quantitative measure of employees' global perceptions, and the survey with 6-item is about how safety is managed and addressed within their organization. Flin and Mearns [16] defined the "big five" themes of safety climate: management, safety system, risk, work pressure, and competence. Glendon and Litherland [18] examined the safety climate in a road construction organization and found six factors: communication and support, adequacy of procedures, work pressure, personal protective equipment, relationships, and safety rules. Similarly, two other related studies [8] identified 10 similar items of safety climate on construction sites. These cover the broad areas of organizational context, social environment, individual appreciation, and work environment.

According to the reviews of previous studies and the actual situation, this paper takes the definition that safety climate refers only to the workers' perception on the value of safety in the work environment, which is at the level of organization. The safety climate in this paper is mainly divided into three dimensions, including managers' attention, project safety environment, and safety supervision with seven items.

2.2. Research Hypotheses. The organizational safety climate defines the perception of the value of safety in the work environment, which in turn affects individual behavior. In order to address the effect that the safety climate at the organizational level has on the individual awareness and behavior, and the relationship between them, the following hypotheses are developed.

Safety awareness refers to an "individual's own awareness of safety issues" [19]. This awareness works on both a cognitive and behavioral level. Cognitively, safety awareness means being mentally aware of safety in one's work and recognizing what behaviors foster operational safety. 
Behaviorally, safety awareness encourages the behavior that fosters operational safety. Therefore, safety behavior is the employee's immediate emotional response of the awareness.

The present study has examined the relationship between safe behavior and safety awareness. Choi et al. [3] believe that increased self-awareness could improve construction workers' safety behavior. The analysis results of the investigation by Sun et al. [20] indicate that safety awareness has direct and positive influence on the safety behavior. The influence of awareness on behavior is mainly based on "the attitude-behavior theory," and Mills [21] pointed out that what really impacts the behavior of the individual is not the objective-specific conditions or events, but the way in which information is being interpreted and processed by the individual. Specifically, it could be concluded that one's attitude will affect one's behaviors; that is, the safety awareness could affect the safety behavior. This leads to Hypothesis 1.

\subsubsection{Hypothesis 1. Safety awareness has a significant} positive effect on safety behavior.

(1) The Safety Climate and Safety Behavior. Previous research revealed a positive association between safety climate and seafarers' safety behavior [22-24]. Some research proved that safety climate is positively and significantly associated with safety behaviors [25-27]. Seo et al. [28] pointed that safety climate had a significant direct effect on safety behavior. Bronkhorst [11] takes the same opinion that strengthening the safety climate within an organization can increase employees' safety behavior. Fang et al. [8] point out that safety climate can play an important part in increasing safety behavior. Individual perceptions of safety climate exerted a causal effect on individual safety behavior [29], and safety climate was assessed as relating to individual workers' reported safety behavior [30]. Kapp [31] pointed out that under positive group safety climate conditions, employee safety compliance behavior improves. Other research held the opinion that the organizational safety climate can act as an indirect predictor of proactive and compliance safety behaviors [32]. There is relatively little existing research, however, into the mechanisms by which safety climate affects safety behavior. This leads to Hypothesis 2.

2.2.2. Hypothesis 2. Safety climate has a significant positive effect on safety behavior.

(1) The Safety Climate and Safety A wareness. In the literature of psychology, however, the empirical research that examined safety awareness is not sufficient. Safety climate concerns worker perception of safety as related to the organizational environment, whereas safety awareness concerns worker perception of safety as related to individual. Safety climate exists at a higher level and has an impact on safety awareness. In some research, the safety awareness is included in the safety climate as one dimension named the workers' self-perception of safety [14]. The promotion of employees' safety awareness primarily depends on whether there exists a positive safety climate within the construction company [33]. The safety climate (which includes the physical working environment and social influence) is one of the most significant factors affecting safety awareness [18]. A study of safety climate in Serbian industrial workplace presented that safety climate involves safety awareness and competency [34]. This therefore leads to Hypothesis 3.

2.2.3. Hypothesis 3. Safety climate has a significant positive effect on safety awareness.

(1) The Moderating Effect of Safety Climate on Safety Awareness and Safety Behavior. Workers' safety behavior is influenced by perceived management norm, perceived workgroup norm, and personal attitude [3]. Under a positive climate of safety, the employees develop site safety awareness and accept that the first priority during any site operation is safety. Thus, they will take the initiative to adopt practices that will inevitably reduce the violation of safety rules [33]. Safety climate could affect the safety behavior positively through the working environment and safety awareness [20]. In other words, safety climate has a moderating effect on the relationship between safety awareness and safety behavior, that is, when the safety climate in an organization is higher, the safety awareness has a more significant positive effect on safety behavior. So this leads to Hypothesis 4 .

2.2.4. Hypothesis 4. Safety climate has a positive moderating effect on the relationship between individual safety awareness and safety behavior, such that the relationship between safety awareness and safety behavior will be stronger when the safety climate is positive.

Finally, it creates a hierarchical-layer linear model that includes safety climate, safety awareness, and safety behavior. The individual level includes construction worker safety awareness and safety behavior as two variables. The organizational level includes the safety climate variable.

The research model is shown in Figure 2 and provides an overview of these hypotheses.

2.3. Questionnaire. The survey instructions for the questionnaire were designed to ensure all employees, regardless of education level, understood the questions. It comprised four parts: personal information, safety climate, safety awareness, and safety behavior. The scales are constructed using a Likert five-point scaling technique with the survey respondents selecting a number from 1 to 5 as appropriate. The 1-5 scale represents "strongly disagree, little disagree, agree, little agree, and strongly agree," respectively.

Brondino et al. [35] developed Co-workers' Safety Climate (CSC) scale to measure safety climate. The questionnaire developed by Sun et al. [20] is based on the safety climate survey questionnaire of Health and Safety Climate Survey Tool of HSE, United Kingdom. Mearns et al. [7] used the Offshore Safety Questionnaire (OSQ) to conduct the 


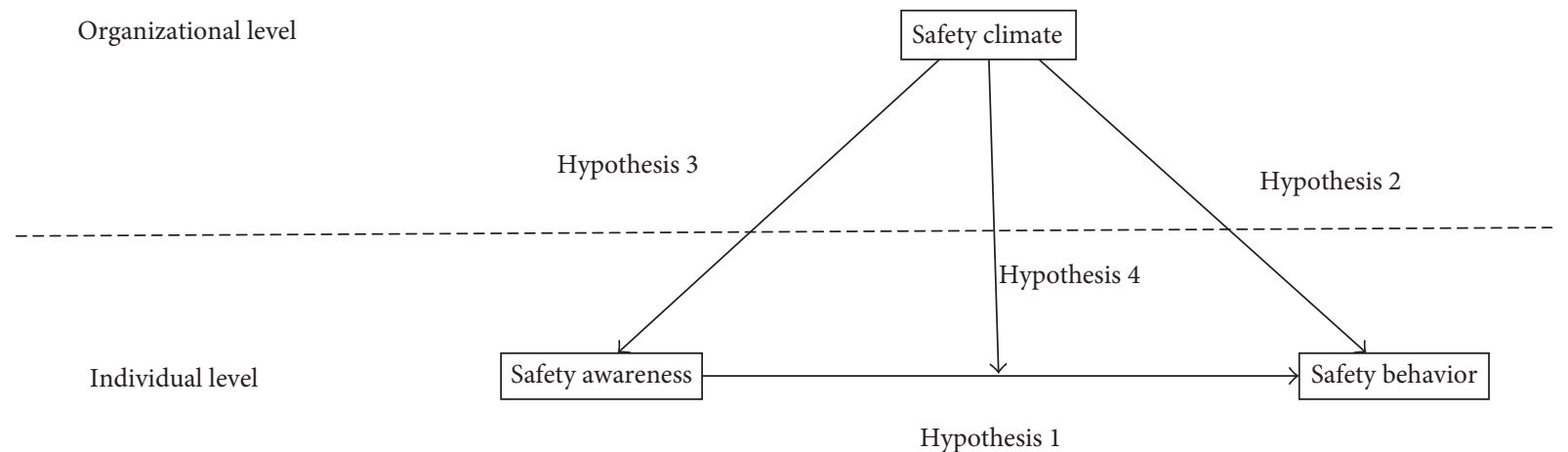

FIgURE 2: Research model and hypotheses.

safety climate survey. The questionnaire items were adapted from the research of Brown and Holmes [36], Sun et al. [20], and Mearns et al. [7], combining with the results of investigation at construction site. This paper used three dimensions to measure safety climate, that is, addressed managers' attention, project safety environment, and safety supervision, which included seven questions.

According to the reviews on the safety awareness, this paper adopted it into three dimensions in the items design of questionnaire [19], including individual safety cognition, safety attitude, and safety participation, which included eight questions.

Neal and Griffin [37] developed safety behavior scale to measure safety behavior, comprising two factors, safety compliance and safety participation. Zhou and Fang [38], Hahn and Murphy [39], Zhang and Zhang [40], and so on adopted safety measures, safety handling, employee safety protection, safety specification observed, and accident rate as measuring variables of safety behavior. The research in this paper mainly focuses on the performance of the safety behavior, which is adopted into two dimensions including the self-reported accident rate and safety specification observation, to design the questionnaire with three questions in total.

2.4. Sample. For this research, a formal survey was conducted by questionnaire from April 15, 2016 to May 18, 2016 among construction workers. In total, 164 questionnaires were received from 15 construction teams, respectively, and 96 of them were valid. Among the 96 respondents, 74 were common workers, 16 were construction team managers, and 6 were temporary workers. Because of the generally low cultural level of construction workers and the need to guarantee the authenticity and accuracy of results, a face-toface survey form was selected for data collection.

The average age of the respondents was 42 . The standard deviation was $7.621(n=96)$, showing a large difference in age and pointing to the universality of the research conclusions. Of the respondents, it revealed from the trend line in Figure 3 that a majority $(62.0 \%)$ of construction worker respondents were between 35 and 50 years old.

The respondents' educational attainment was mainly at middle school (86.0\%) and high school (14.0\%) levels.

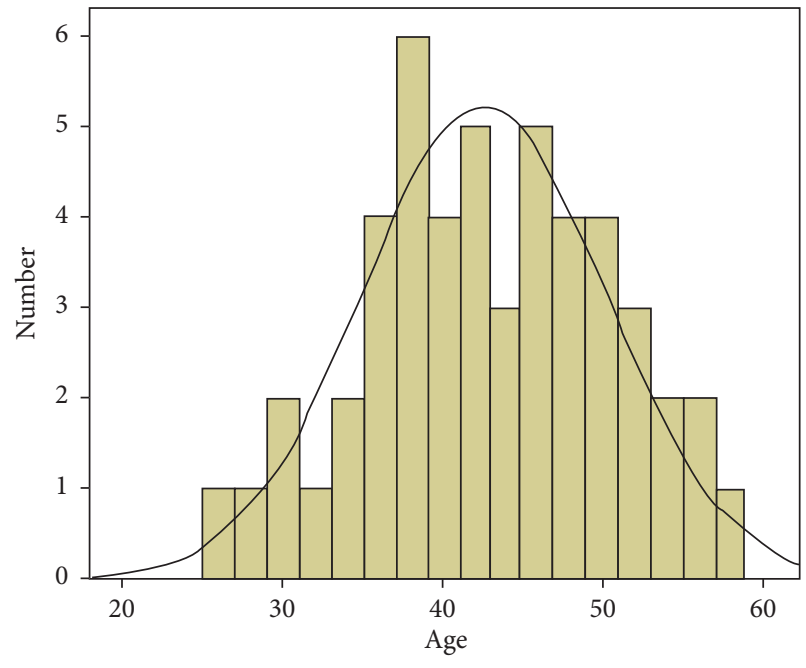

FIGURE 3: Age of participants in the sample.

The above data show that today's construction workers in China have a generally low cultural status.

Respondents having worked from 5 to 10 years accounted for $14.0 \%$ of the total. From 10 to 15 years, it was $4.0 \%$; from 15 to 20 years, $12.0 \%$; from 20 to 25 years, $36.0 \%$; from 25 to 30 years, $18.0 \%$; and more than 30 years, $16.0 \%$. A $36.0 \%$ majority of construction workers had been in the industry between 20 and 25 years.

According to the analysis of worker personal factors analysis on the construction industry's safety climate, it found that the age, position, cultural status, and years of experience of workers will have a certain influence on the construction safety climate. Analysis pointed out that the perception of a construction site environment for temporary workers is lower than that for regular workers. When construction worker educational attainment is higher, the perception of climate is stronger. Safety awareness is lowest for a 35- to 45-year-old worker. For workers less than 35 years old and above the age of 45 , it is higher. Progressively, after the age of 40 , it rises. The number of years at work and the safety climate are positively related to the safety awareness and behavior. With the number of years at work being more than 20, the safety environment perception increases. 
TABLE 1: The scores of safety climate, safety awareness, and safety behavior.

\begin{tabular}{lccccc}
\hline & $N$ & Min & Max & Average & Standard deviation \\
\hline Safety climate & 96 & 16 & 22 & 19.94 & 1.86 \\
Safety awareness & 96 & 24 & 33 & 28.82 & 2.83 \\
Safety behavior & 96 & 5 & 13 & 8.55 & 2.44 \\
\hline
\end{tabular}

\section{Results and Discussion}

3.1. Results. The respondents' scores of safety climate, safety awareness, and safety behavior are illustrated in Table 1 . The scale of safety climate is 35 points ( 7 questions, each counting for 5 points). The highest and lowest scores are 22 and 16, respectively. The overall average score is 19.94 points with a standard deviation of 1.86 . According to data in Table 1, the perception of safety climate among construction workers is not high. Furthermore, significant differences exist among workers. In a word, the construction industry needs to improve its safety climate further.

The scale of safety awareness is 40 points ( 8 questions, each counting for 5 points). The highest and lowest scores are 33 and 24, respectively. The overall average score is 28.82 points with a standard deviation of 2.83. Data in Table 1 show that construction workers have a relatively strong sense of safety awareness, but a standard deviation greater than 2 indicates a disparate level of safety awareness among workers in the construction industry.

The total score of construction worker safety behavior is 15 points. Data from Table 1 show that safety behavior among construction workers scored an average of 8.55, the highest being 13 and the lowest 5 . Low safety behavior scores mean unsafe behavior is common in construction teams. At the same time, the standard deviation is 2.44 . This shows significant variations in safety behavior among construction workers.

3.2. Empirical Research. The collected quantitative data were further analyzed using HLM, which is also referred to as multilevel analysis or random coefficient models [41, 42]. It is a means of handling nested data statistics. By defining different levels (layers) of model, the random variation is decomposed into two parts. One is the error from the first level individual differences. The other is from the second level organizational differences. HLM has a complete model containing predictor variables in the first layer and prediction variables in second layer. They explain in theory how the entire dependent variable mutation can be affected by the factors in layer 1 and layer 2. They also explain how other models like the null model, random effects model, regression model, and development model are derived from research carried out on the full model.

3.2.1. Null Model. This research firstly established a null model to run the variance component analysis mainly used to observe dependent variables in construction at
TABle 2: Null model.

\begin{tabular}{lccccc}
\hline Random effect & $\begin{array}{c}\text { Standard } \\
\text { deviation }\end{array}$ & $\begin{array}{c}\text { Variance } \\
\text { component }\end{array}$ & d.f. & $\chi 2$ & $P$ value \\
\hline INTRCPT1, u0 & 2.06982 & 4.28415 & 14 & 213.98111 & $<0.001$ \\
Level 1, $r$ & 1.38250 & 1.91132 & & & \\
\hline
\end{tabular}

organization level and check for any statistically significant difference. The model is built in two levels:

$$
\begin{aligned}
\text { Level 1: } \mathrm{SB}_{i j} & =\beta_{0 j}+r_{i j}, \\
\text { Level 2: } \beta_{0 j} & =\gamma_{00}+U_{0 j},
\end{aligned}
$$

where $\mathrm{SB}=$ safety behavior, $\beta_{0 j}=$ the mean value in safety climate for group $j, \gamma_{00}=$ the total mean value in safety climate, variance $\left(r_{i j}\right)=$ within-group variance in safety climate, and variance $\left(U_{0 j}\right)=$ between-group variance in safety climate.

The results of null model analysis are shown in Table 2.

The standard deviation and corresponding chi-square of estimate results all reached the significant level $(P<0.001)$ as seen in Table 2. The construction organization variation can explain $67 \%$ of the total variation in the level of construction worker safety behavior problems. So it is very necessary to build a HLM model.

3.2.2. Hypothesis Testing Using HLM. To find the relationship between safety climate, safety awareness, and safety behavior, we build four models based on the hypotheses using HLM.

(1) Testing of Hypothesis 1. In order to test Hypothesis 1, introduce the variable of safety awareness $\left(\mathrm{SA}_{i j}\right)$ into Level 1 and then build the model.

$$
\begin{aligned}
\text { Level 1: } \mathrm{SB}_{i j} & =\beta_{0 j}+\beta_{1 j}\left(\mathrm{SA}_{i j}\right)+r_{i j}, \\
\text { Level 2: } & \beta_{0 j}=\gamma_{00}+U_{0 j}, \\
\beta_{1 j} & =\gamma_{10}+U_{1 j},
\end{aligned}
$$

where $\mathrm{SA}=$ safety awareness, $\beta_{0 j}=$ the intercept value for group $j, \beta_{1 j}=$ the regression slope for group $j, \gamma_{00}=$ mean of the intercepts across groups, $\gamma_{10}=$ mean of the slopes across groups (Hypothesis 1), variance $\left(r_{i j}\right)=$ Level 1 residual variance, variance $\left(U_{0 j}\right)=$ variance in intercepts, and variance $\left(U_{1 j}\right)=$ variance in slopes.

In this model, $\gamma_{00}$ and $\gamma_{10}$ represent the average coefficient of $\beta_{0 j}$ and $\beta_{1 j}$ in Level 1 , respectively, and $\gamma_{10}$ represents the relationship between safety awareness and safety behavior across construction groups, which can be used to test Hypothesis 1. Analysis results are as follows: $\gamma_{10}=0.48$, $P<0.1$. This shows that safety awareness for construction workers has a positive influence on safety behavior. The $t$-test results reach a statistically significant level, so Hypothesis 1 is supported.

(2) Testing of Hypothesis 2. In order to test Hypothesis 2, introduce the variable of safety climate $\left(\mathrm{SC}_{j}\right)$ into Level 2 and then build the model. 
TABLE 3: HLM test on hypotheses.

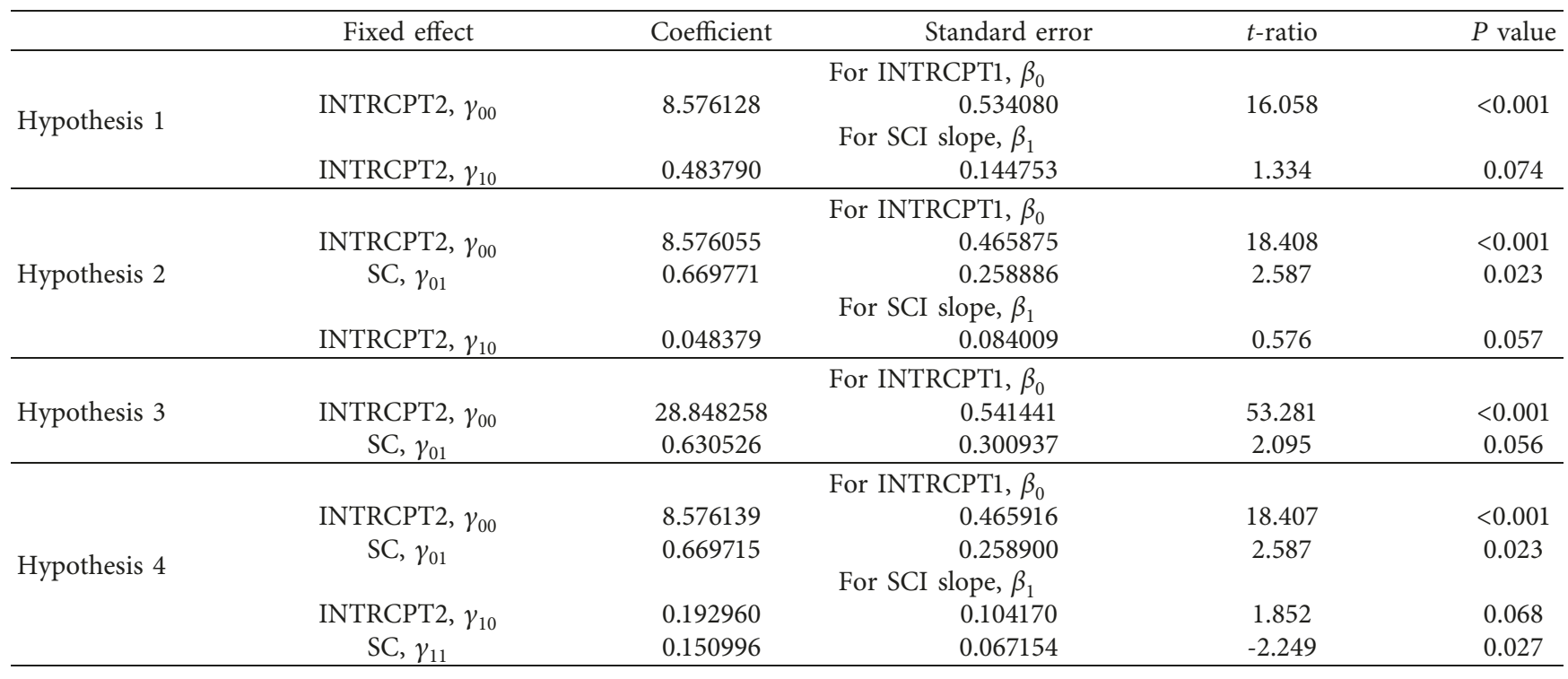

Level 1: $\mathrm{SB}_{i j}=\beta_{0 j}+\beta_{1 j}\left(\mathrm{SA}_{i j}\right)+r_{i j}$,

Level 2: $\beta_{0 j}=\gamma_{00}+\gamma_{01}\left(\mathrm{SC}_{j}\right)+U_{0 j}$,

$$
\beta_{1 j}=\gamma_{10}+U_{1 j}
$$

where $\mathrm{SC}=$ safety climate, $\gamma_{00}=$ intercept of Level 2 regression predicting $\beta_{0 j}, \gamma_{01}=$ slope of Level 2 regression predicting $\beta_{0 j}$ (Hypothesis 2), $\gamma_{10}=$ intercept of Level 2 regression predicting $\beta_{1 j}$, variance $\left(r_{i j}\right)=$ variance in Level 1 residual, variance $\left(U_{0 j}\right)=$ variance in Level 2 residual for models predicting $\beta_{0 j}$, and variance $\left(U_{1 j}\right)=$ variance in Level 2 residual for models predicting $\beta_{1 j}$;

In the above model, the relationship between safety climate and safety behavior across construction teams by controlling safety awareness in Level 1 is represented. A $t$-test on $\gamma_{01}$ is used to test hypothesis 2 . The analysis results are as follows: $\gamma_{10}=0.67, P<0.1$. This shows that the safety climate for construction workers has a significant positive influence on safety behavior. The $t$-test results reach a statistically significant level, so Hypothesis 2 is supported.

(3) Testing of Hypothesis 3. Model building for Hypothesis 3.

$$
\begin{aligned}
& \text { Level 1: } \mathrm{SA}_{i j}=\beta_{0 j}+r_{i j} \\
& \text { Level 2: } \beta_{0 j}=\gamma_{00}+\gamma_{01}\left(\mathrm{SC}_{j}\right)+U_{0 j},
\end{aligned}
$$

where $\gamma_{00}=$ intercept of Level 2 regression predicting $\beta_{0 j}$, $\gamma_{01}=$ slope of Level 2 regression predicting $\beta_{0 j}(\mathrm{Hy}-$ pothesis 3$)$, variance $\left(r_{i j}\right)=$ variance in Level 1 residual, and variance $\left(U_{0 j}\right)=$ variance in Level 2 residual for models predicting $\beta_{0 j}$.

In the above model, the $t$-test can be used to test Hypothesis 3. Analysis results are as follows: $\gamma_{10}=0.63$, $P<0.1$. This shows that the safety climate for construction workers has a significant positive influence on safety awareness. The $t$-test results reach a statistically significant level, so Hypothesis 3 is supported.

(4) Testing of Hypothesis 4. Model building for Hypothesis 4.

$$
\begin{aligned}
\text { Level 1: } & \mathrm{SB}_{i j}=\beta_{0 j}+\beta_{1 j}\left(\mathrm{SA}_{i j}\right)+r_{i j}, \\
\text { Level 2: } & \beta_{0 j}=\gamma_{00}+\gamma_{01}\left(\mathrm{SC}_{j}\right)+U_{0 j}, \\
\beta_{1 j} & =\gamma_{10}+\gamma_{11}\left(\mathrm{SC}_{j}\right)+U_{1 j},
\end{aligned}
$$

where $\gamma_{00}=$ intercept of Level 2 regression predicting $\beta_{0 j}, \gamma_{01}=$ slope of Level 2 regression predicting $\beta_{0 j}, \gamma_{10}=$ intercept of Level 2 regression predicting $\beta_{1 j}, \gamma_{11}=$ slope of Level 2 regression predicting $\beta_{1 j}$ (Hypothesis 4 ), variance $\left(U_{0 j}\right)=$ variance in Level 2 residual for models predicting $\beta_{0 j}$, and variance $\left(U_{1 j}\right)=$ variance in Level 2 residual for models predicting $\beta_{1 j}$.

In the above model, $\gamma_{11}$ represents the estimate of interaction between safety climate and safety awareness, and the $t$-test on $\gamma_{11}$ can be used to test hypothesis 4 . Analysis results are as follows: $\gamma_{11}=0.15, P<0.1$. This shows that safety climate positively adjusts the influence of the safety awareness of safety behavior. Furthermore, $t$-test results reach a statistically significant level, so Hypothesis 4 is supported.

3.2.3. HLM Test on Hypotheses. The parameters of each model are shown in Table 3; based on the analyses above, it can be conclude that the four hypotheses are all supported.

\section{Discussion and Conclusion}

From the analysis of hypotheses above, it is obvious that the safety climate of construction workers has a significant positive influence on safety behavior as influence coefficient is 0.67 . And the safety climate also has a positive influence on safety awareness as influence coefficient is 0.63 . It can be 
analyzed that the factor at organizational level can influence the factors at the individual level.

At the individual level, it is analyzed that the safety awareness of construction workers has a positive influence on safety behavior, with an influence coefficient of 0.48 . What is more, a positive safety climate could strengthen the effect of construction worker safety awareness on safety behavior with an influence coefficient of 0.15 . The safety behavior scores 8.55 points out of 15 on average, which indicates that the present safety level of construction worker behavior is unsatisfied. Therefore, strengthening the construction safety climate and safety awareness becomes a priority in the effort to reduce unsafe behavior among construction workers.

In conclusion, according to the results of hierarchical linear analysis, the four hypotheses are all supported, which means improving the individual safety awareness and the organizational safety climate could ultimately reduce unsafe behavior and accordingly make contribution to safety management in construction. A positive safety climate will make contribution to the individual safety awareness and behavior and then strengthen the relationship between them. As a result, it can be concluded that the safety management should not only concentrate on the individual behavior and awareness but also take safety climate into consideration. For instance, strengthening the leaders' attention to safety, improving the construction project's safety environment and the project safety supervision mechanism, and training and cultivating safety attitudes and participation are also helpful to reducing unsafe behavior of workers.

\section{Data Availability}

The detailed data of survey results used to support the findings of this study are available from the corresponding author upon request.

\section{Conflicts of Interest}

The authors declare that they have no conflicts of interest.

\section{References}

[1] M. Y. Leung, Q. Liang, and P. Olomolalye, "Impact of job stressors and stress on the safety behavior and accidents of construction workers," Journal of Engineering and Technology Management, vol. 10, no. 1061, pp. 1943-5479, 2015.

[2] Y. Feng, E. A. L. Teo, F. Y. Y. Ling, and S. P. Low, "Exploring the interactive effects of safety investments, safety culture and project hazard on safety performance: an empirical analysis," International Journal of Project Management, vol. 32, no. 6, pp. 932-943, 2014.

[3] B. Choi, S. Ahn, and S. H. Lee, "Role of social norms and social identifications in safety behavior of construction workers. I: theoretical model of safety behavior under social influence," Journal of Construction Engineering and Management, vol. 10, no. 1061, pp. 1943-7862, 2016.

[4] T. Niskanen, "Safety climate in the road administration," Safety Science, vol. 7, pp. 237-255, 1994.

[5] M. A. Griffin and A. Neal, "Percetions of safety at work: a framework for linking safety climate to safety performance, knowledge, and motivation," Journal of Occupational Health Psychology, vol. 5, no. 3, pp. 347-358, 2000.

[6] S. Cox and R. Flin, "Safety culture: philosopher's stone or man of straw?,"Work and Stress, vol. 12, no. 3, pp. 189-201, 1998.

[7] K. Mearns, S. M. Whitaker, and R. Flin, "Safety climate, safety management practice and safety performance in offshore environments," Safety Science, vol. 41, no. 8, pp. 641-680, 2003.

[8] D. Fang, Y. Chen, and L. Wong, "Safety climate in construction industry: a case study in Hong Kong," Journal of Construction Engineering and Management, vol. 132, no. 6, pp. 573-584, 2006.

[9] E. A. L. Teo and Y. Feng, "The role of safety climate in predicting safety culture on construction sites," Architectural Science Review, vol. 52, no. 1, pp. 5-16, 2009.

[10] M. D. Cooper and R. A. Phillips, "Exploratory analysis of the safety climate and safety behavior relationship," Journal of Safety Research, vol. 35, no. 5, pp. 497-512, 2004.

[11] B. Bronkhorst, "Behaving safely under pressure: the effects of job demands, resources, and safety climate on employee physical and psychosocial safety behavior," Journal of Safety Research, vol. 55, pp. 63-72, 2015.

[12] M. A. Idris, M. F. Dollard, J. Coward, and C. Dormann, "Psychosocial safety climate: conceptual distinctiveness and effect on job demands and worker psychological health," Safety Science, vol. 50, pp. 19-28, 2012.

[13] J. Bosak, W. J. Coetsee, and S. J. Cullinane, "Safety climate dimensions as predictors for risk behavior," Accident Analysis and Prevention, vol. 55, no. 6, pp. 256-264, 2013.

[14] Q. Li, C. Ji, J. Yuan, and R. Han, "Developing dimensions and key indicators for the safety climate within China's construction teams: a questionnaire survey on construction sites in Nanjing," Safety Science, vol. 93, pp. 266-276, 2017.

[15] B. Fernández-Muñiz, J. M. Montes-Peón, and C. J. VázquezOrdás, "Safety climate in OHSAS 18001-certified organizations: antecedents and consequences of safety behavior," Accident Analysis and Prevention, vol. 45, no. 2, pp. 745-758, 2012.

[16] R. Flin and K. Mearns, "Measuring safety climate: identifying the common features," Safety Science, vol. 34, no. 1-3, pp. 177-192, 2000.

[17] D. Zohar and G. Luria, "Multilevel model of safety climate: cross-level relationships between organization and grouplevel climates," Journal of Applied Psychology, vol. 9, no. 4, pp. 616-628, 2005.

[18] A. I. Glendon and D. K. Litherland, "Safety climate factors, group differences and safety behavior in road construction," Safety Science, vol. 39, no. 3, pp. 157-188, 2001.

[19] F. Kiani and M. R. Khodabakhsh, "Promoting individual learning for trainees with perceived high helplessness: experiences of a safety training program," Iranian Journal of Health Sciences, vol. 8, no. 4, pp. 19-28, 2014.

[20] J. Sun, S. Yan, and C. Y. Du, "Empirical study on the effect of safety atmosphere on the safety performance in the construction enterprises," Journal of Safety and Environment, vol. 14, no. 2, pp. 60-64, 2014, in Chinese.

[21] J. H. Mills, Making Sense of Organizational Change, Routledge, London, UK, 2003.

[22] A. Cheyne, J. M. Tomás, and A. Oliver, "Multilevel models in the explanation of the relationship between safety climate and safe behavior," Spanish Journal of Psychology, vol. 16, no. 2, pp. 1-11, 2013.

[23] X. Liu, G. Huang, H. Huang, S. Wang, Y. Xiao, and W. Q. Chen, "Safety climate, safety behavior, and worker 
injuries in the Chinese manufacturing industry," Safety Science, vol. 78, no. 6, pp. 173-178, 2015.

[24] C. S. Lu and C. L. Tsai, "The effect of safety climate on seafarers' safety behaviors in container shipping," Accident Analysis and Prevention, vol. 42, no. 6, pp. 1999-2006, 2010.

[25] N. V. Schwatka and J. C. Rosecrance, "Safety climate and safety behaviors in the construction industry: the importance of co-workers commitment to safety," Work, vol. 54, no. 2, pp. 401-413, 2016.

[26] T. D. Smith, F. Eldridge, and D. M. Dejoy, "Safety-specific transformational and passive leadership influences on firefighter safety climate perceptions and safety behavior outcomes," Safety Science, vol. 86, pp. 92-97, 2016.

[27] G. D. Kearney, G. Rodriguez, S. A. Quandt, J. T. Arcury, and T. A. Arcury, "Work safety climate, safety behaviors, and occupational injuries of youth farmworkers in North Carolina," American Journal of Public Health, vol. 105, no. 7, pp. 1336-1343, 2015.

[28] H. C. Seo, Y. S. Lee, J. J. Kim, and N. Y. Jee, “Analyzing safety behaviors of temporary construction workers using structural equation modeling," Safety Science, vol. 77, pp. 160-168, 2015.

[29] S. L. Tholén, A. Pousette, and M. Törner, "Causal relations between psychosocial conditions, safety climate and safety behavior-a multi-level investigation," Safety Science, vol. 55, no. 55 , pp. $62-69,2013$.

[30] S. L. Morrow, A. K. Mcgonagle, M. Dove-Steinkamp, C. T. Walker, M. Marmet, and J. Barnes-Farrell, "Relationships between psychological safety climate facets and safety behavior in the rail industry: a dominance analysis," Accident Analysis and Prevention, vol. 42, no. 5, pp. 14601467, 2010.

[31] E. A. Kapp, "The influence of supervisor leadership practices and perceived group safety climate on employee safety performance," Safety Science, vol. 50, no. 4, pp. 1119-1124, 2012.

[32] C. S. Fugas, S. A. Silva, and J. L. Meliá, "Another look at safety climate and safety behavior: deepening the cognitive and social mediator mechanisms," Accident Analysis and Prevention, vol. 45, no. 1, pp. 468-477, 2012.

[33] Q. Zhou, D. Fang, and S. Mohamed, "Safety climate improvement: case study in a Chinese construction company," Journal of Construction Engineering and Management, vol. 137, no. 1, pp. 86-95, 2011.

[34] N. Milijic, I. Mihajlovic, N. Strbac, and Z. Zivkovic, "Developing a questionnaire for measuring safety climate in the workplace in Serbia," International Journal of Occupational Safety and Ergonomics, vol. 19, no. 4, pp. 631-645, 2013.

[35] M. Brondino, S. A. Silva, and M. Pasini, "Multilevel approach to organizational and group safety climate and safety performance: co-workers as the missing link," Safety Science, vol. 50, no. 9, pp. 1847-1856, 2011.

[36] R. L. Brown and H. Holmes, "The use of a factor-analytic procedure for assessing the validity of an employee safety climate model," Accident Analysis and Prevention, vol. 18, no. 6, pp. 455-470, 1986.

[37] A. Neal and M. A. Griffin, "A study of the lagged relationships among safety climate, safety motivation, safety behavior, and accidents at the individual and group levels," Journal of Applied Psychology, vol. 91, no. 4, pp. 946-953, 2006.

[38] Q. Zhou and D. Fang, "An empirical study on the influence mechanism of safety climate on safety behavior in construction industry," China Civil Engineering Journal, vol. 11, pp. 129-132, 2009, in Chinese.
[39] S. E. Hahn and L. R. Murphy, "A short scale for measuring safety climate," Safety Science, vol. 46, no. 7, pp. 1047-1066, 2008.

[40] J. G. Zhang and L. Zhang, "Study on the impact of safety climate on enterprise safety behavior," Journal of Safety Science and Technology, vol. 3, no. 1, pp. 106-110, 2007, in Chinese.

[41] Y. Matsuyama, Hierarchical Linear Modeling (HLM), Springer, New York, NY, USA, 2013.

[42] D. A. Hofmann, M. A. Griffin, and M. B. Gavin, "The application of hierarchical linear modeling to organizational research," in Multilevel Theory, Research, and Methods in Organizations: Foundations, Extensions, and New Directions, K. J. Klein and W. J. Kozlowski, Eds., pp. 467-511, JosseyBass, San Francisco, CA, USA, 2000. 


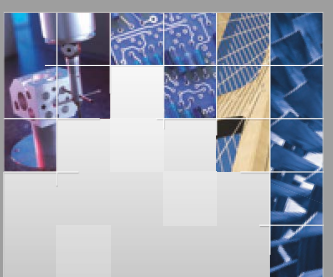

\section{Enfincering}
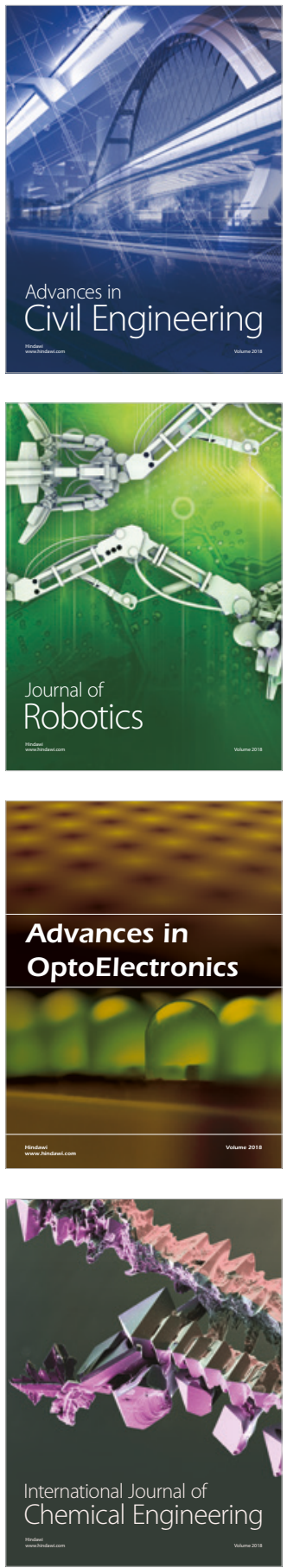

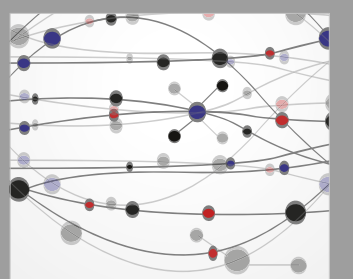

\section{Rotating \\ Machinery}

The Scientific World Journal

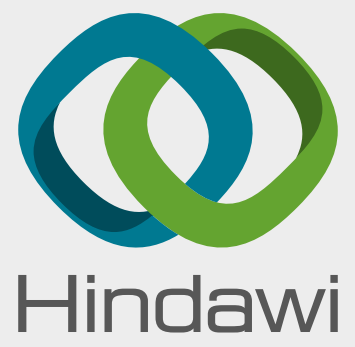

Submit your manuscripts at

www.hindawi.com
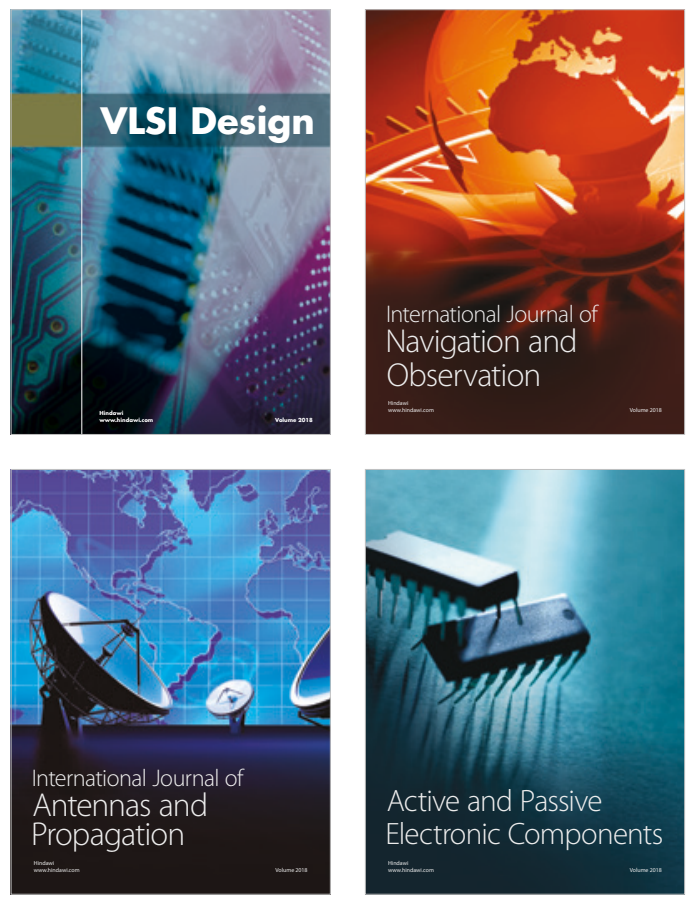
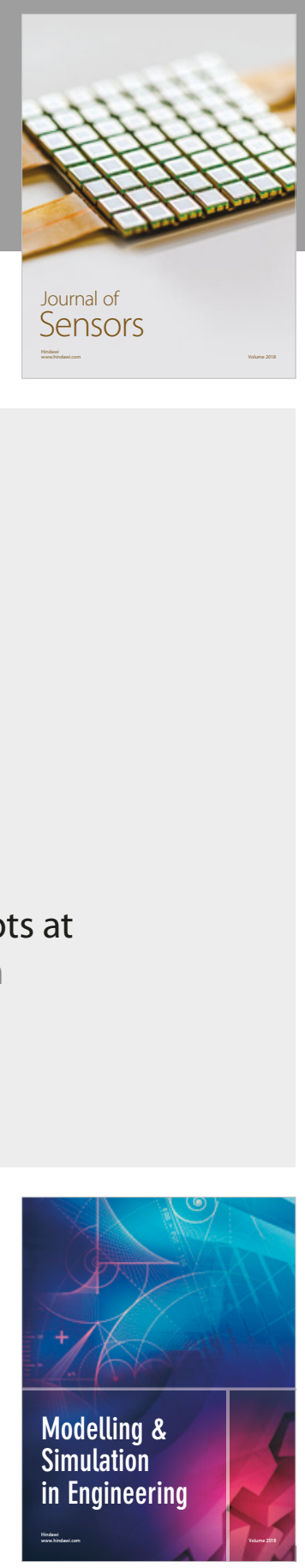

\section{Advances \\ Multimedia}
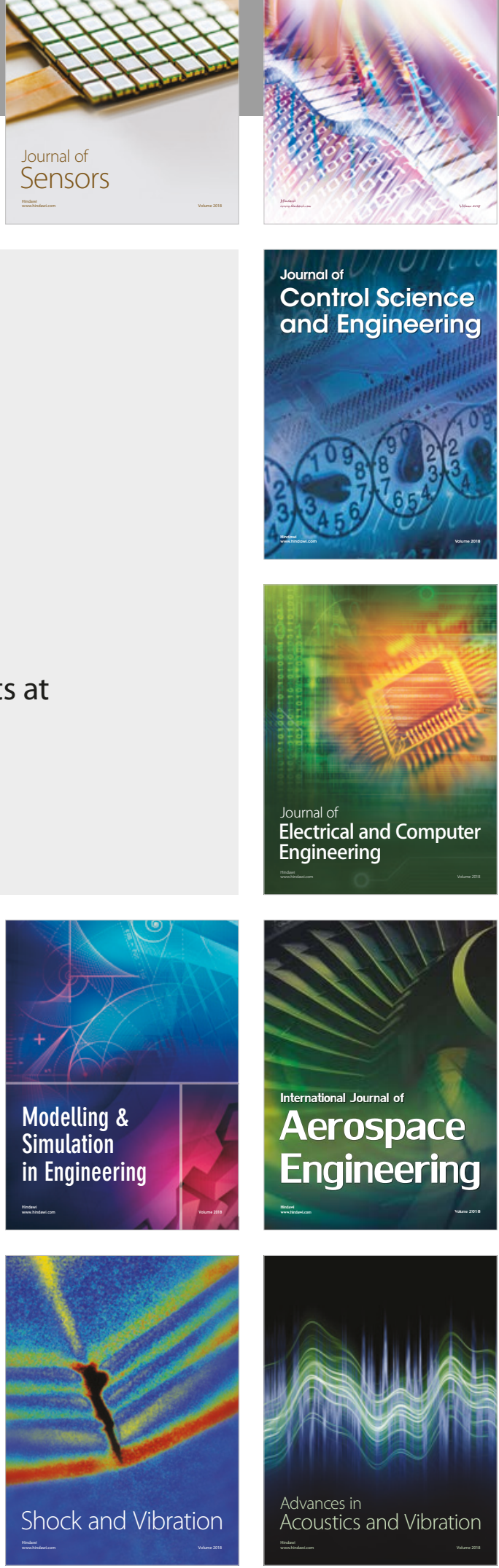